\title{
Alpha-fetoprotein-L3 and Golgi protein 73 may serve as candidate biomarkers for diagnosing alpha- fetoprotein-negative hepatocellular carcinoma
}

This article was published in the following Dove Press journal:

OncoTargets and Therapy

31 December 20I5

Number of times this article has been viewed

\author{
Zhiguo Zhang' \\ Yanying Zhang ${ }^{2}$ \\ Yeying Wang' \\ Lingling $X u^{3}$ \\ Wanju $\mathrm{Xu}^{3}$
}

'Department of Clinical Laboratory, Zhangqiu Maternity and Child Care Hospital, Zhangqiu, ${ }^{2}$ Department of Clinical Laboratory, Zaozhuang City Wangkai Infection Hospital, Zaozhuang, ${ }^{3}$ Department of Clinical Laboratory, Qianfoshan Hospital, Jinan, People's Republic of China
Correspondence: Wanju Xu

Department of Clinical Laboratory,

Qianfoshan Hospital, Jingshi Road,

No 16766, Jinan 2500I4, People's

Republic of China

Tel +86I379I07 7359

Email tksxfd@।63.com
Abstract: Currently, there is no reliable biomarker for use in diagnosing alpha-fetoprotein (AFP)-negative hepatocellular carcinoma (HCC). Such a biomarker would aid in making an early diagnosis of AFP-negative HCC, ensuring the timely initiation of treatment. This study examined AFP-L3 and Golgi protein 73 (GP73) as candidate biomarkers for AFP-negative HCC. The affinity adsorption method and enzyme-linked immunoassays were separately used to determine serum levels of AFP-L3 and GP73 in 50 patients with AFP-negative HCC, 30 non-HCC patients, and 50 healthy subjects. Fifty percent of patients with AFP-negative HCC tested positive for AFP-L3, while $3.33 \%$ of non-HCC patients and $2.00 \%$ of healthy subjects were AFP-L3 positive. Patients with AFP-negative HCC had significantly higher serum levels of AFP-L3 compared to non-HCC patients and healthy individuals; however, there was no significant difference in the AFP-L3 levels of non-HCC patients and healthy subjects. Sixty-six percent of patients with AFP-negative HCC tested positive for GP73, while 10\% of non-HCC patients and $0 \%$ of healthy subjects were GP73-positive. Patients with AFP-negative HCC had significantly higher serum levels of GP73 compared to non-HCC patients and healthy subjects, but there was no significant difference between the GP73 levels of non-HCC patients and healthy individuals. Moreover, 20 patients with AFP-negative HCC were both AFP-L3- and GP73-positive, while no non-HCC patients or healthy subjects tested positive for both markers. Either AFP-L3 or GP73 may be used as a biomarker for diagnosing AFP-negative HCC, while their combined use provides improved diagnostic accuracy and greater sensitivity.

Keywords: liver cancer, AFP-negative HCC, AFP-L3, GP73, AFP

\section{Introduction}

Hepatocellular carcinoma (HCC), the incidence of which has been gradually increasing, is the fifth most common malignancy worldwide and the third most common type of cancer in the People's Republic of China. ${ }^{1,2}$ Moreover, it is estimated that $7.18 \%$ of the entire Chinese population is infected with hepatitis B virus (HBV). ${ }^{3,4}$ Until now, alphafetoprotein (AFP)-negative HCC has been difficult to diagnose, and there has been a lack of efficient and reliable diagnostic methods. AFP is a serum protein found at very low concentrations in the sera of normal humans, but is present at high concentrations in sera obtained from newborn humans. While AFP has been widely investigated as a biomarker for diagnosing $\mathrm{HCC}$, it has proven to be a suboptimal diagnostic marker for HCC surveillance, as only a small proportion of individuals with HCC present with elevated AFP levels. AFPs comprise a group of heterogeneous glycoproteins and phytolectins, which contain different sugar moieties and display various features. One specific type of AFP, AFP-L3, binds to lectin Lens culinaris agglutinin and displays 
serum levels which are inconsistent with levels of AFP in human sera. ${ }^{5}$ Furthermore, the AFP-L3 isoform appears to be more specific than the total AFP level for diagnosing HCC. ${ }^{6}$ A retrospective study conducted by Shiraki et al $^{7}$ demonstrated that nine $(41 \%)$ of 21 liver cancer patients showed high concentrations of AFP-L3 at 12 months prior to an imaging diagnosis and that the proportion of AFP-L3 in total AFP was independent of the serum level of total AFP. ${ }^{8}$ If serum levels of AFP-L3 are highly specific for HCC, they could be used to screen individuals at high risk for HCC to achieve its early diagnosis and timely initiation of treatment.

Golgi protein 73, also called GP73, is a Golgi type II transmembrane protein. Kladney et $\mathrm{al}^{9}$ first described GP73 expression in biliary epithelial cells of normal livers, but found it was rarely expressed in hepatocytes. It has also been reported that GP73 expression is upregulated in $\mathrm{HBV}$ or $\mathrm{HCV}$ infection, alcoholic liver disease, or autoimmune hepatitis, and is found at strikingly high serum concentrations in $\mathrm{HCC}$ patients. ${ }^{10}$ Moreover, serum concentrations of GP73 in HCC patients are significantly higher than those in patients with liver cirrhosis. ${ }^{11}$ The sensitivity and accuracy of GP73 as indicators of HCC were shown to be $69 \%$ and $75 \%$, respectively, and the area under the receiver operator characteristic (ROC) curve was greater than that of AFP (0.79 vs 0.61 ). Additionally, GP73 showed greater sensitivity than AFP for detecting early-stage HCC and a 57\% sensitivity for detecting AFP-negative HCC, suggesting its value as a diagnostic marker for early-stage and/or AFP-negative HCC. ${ }^{12}$ A study of 535 patients with HCC or other chronic liver diseases found that all patients had high levels of GP73, ${ }^{13}$ suggesting its usefulness as a biomarker for early-stage HCC.

\section{Materials and methods Study populations}

Between September 2013 and June 2014, we recruited 50 AFP-negative HCC patients from the Qianfoshan Hospital in Shandong province to participate in this study. The inclusion criteria were as follows: 1) confirmed as primary HCC by color Doppler flow imaging or the results of a pathology examination and 2) an AFP level $<8.04 \mu \mathrm{g} / \mathrm{L}$. Additionally, 30 patients ( 23 men and seven women; mean age 47 years, age range from 25 to 71) with other types of liver disorders (22 cases of cirrhosis, seven cases of chronic hepatitis B, and one case of severe hepatitis) were recruited from the same hospital. The diagnostic criteria used for chronic hepatitis B were as follows: 1) hepatitis B confirmed by pathology; 2) thickening of the spleen shown on imaging; and 3) a reversed albumin/globulin ratio. The diagnostic criteria for severe hepatitis included a plasma thromboplastin antecedent $\leq 40 \%$ and a serum total bilirubin level greater than tenfold normal. Liver cirrhosis was confirmed by B-mode ultrasound pathology findings. A list of the criteria used for diagnosing various types of liver disease is shown in Tables 1 and 2. Finally, we recruited a group of 50 healthy subjects (31 men and 19 women; mean age 42 years; range 20 to 68 years) from the same hospital to serve as controls. The 50 healthy subjects were required to meet the following criteria: 1) seronegative for AFP; and 2) no evidence of hepatitis, liver cirrhosis, a liver cyst, cholecyst, or a benign or malignant tumor. The protocol for this study was approved by the Qianfoshan Hospital Ethics Committee (NCT00000789), and all subjects provided a signed informed consent prior to enrollment.

\section{Chemicals and instruments}

The commercial kits used for detecting AFP-L3 and GP73 were purchased from Hotgen Biotech (Beijing, People's Republic of China). AFP detection kits and an ARCHITECT i2000SR automated chemiluminescence immunoassay analyzer were purchased from Abbott Laboratories (Abbott Park, IL, USA). A Zenyth 340 ELISA was purchased from Biochrom (Cambridge, UK).

\section{Sampling}

A $4 \mathrm{~mL}$ sample of fasting venous blood was collected from each subject and stored at room temperature for 1 hour. After classification and labeling, the samples were transferred to storage at $-20^{\circ} \mathrm{C}$.

\section{Detection of AFP-L3, GP73, and AFP}

The affinity adsorption method and immunosorbent assays were separately employed to detect the serum levels of AFP-L3 and GP73. AFP was detected by chemiluminescence. All detection methods were performed in strict accordance with instructions provided by the manufacturer of the detection kit. When using the kits, the critical values for AFP and GP73 were 8.04 and $55 \mu \mathrm{g} / \mathrm{L}$, respectively, and the critical ratio for AFP-L3 as a percentage of total AFP was $10 \%$.

\section{Statistical analysis}

All statistical analyses were performed using GraphPad Prism 5.0 (GraphPad Software Inc., La Jolla, CA, USA). Comparisons of AFP-L3- and GP73-positive rates between various groups were performed using the $\chi^{2}$ test. The sensitivity and accuracy of AFP-L3 and GP73 for detecting AFP-negative HCC were assessed by ROC curves. The confidence level was $95 \%$. 
Table I Criteria used for diagnosing AFP-negative HCC in 50 patients

\begin{tabular}{|c|c|c|c|c|}
\hline Patient & Sex & Age & Admitting diagnosis & $\begin{array}{l}\text { Percussion pain } \\
\text { in hepatic region }\end{array}$ \\
\hline I & Male & 58 & Detected hepatic lesions after 2 days of physical examinations & No \\
\hline 2 & Male & 63 & Pruritus for 6 months; right abdominal pain and swelling & Mild \\
\hline 3 & Male & 62 & Hepatic lesions and enlargement for 2.5 months & No \\
\hline 4 & Male & 48 & Hepatic lesions for 0.5 month & No \\
\hline 5 & Male & 35 & Right abdominal pain for 10 days & Yes \\
\hline 6 & Male & 48 & Right abdominal pain, swelling, nausea, and vomiting for $>I$ month & Mild \\
\hline 7 & Male & 32 & Hepatic lesions detected after 7 days of physical examinations & No \\
\hline 8 & Male & 41 & $\begin{array}{l}\text { Repeated abnormal liver function for } 13 \text { years; abdominal swelling } \\
\text { for } 0.5 \text { month }\end{array}$ & No \\
\hline 9 & Male & 60 & $\begin{array}{l}\text { Chronic hepatitis } B \text { for } 10 \text { years; hepatic lesions detected after } \\
7 \text { days of physical examinations }\end{array}$ & No \\
\hline 10 & Male & 43 & Physical examination revealed right hepatic lesions & No \\
\hline 11 & Male & 77 & Chest pain for 10 days & Yes \\
\hline 12 & Male & 56 & Lower extremity edema and hypodynamia for $>10$ days & No \\
\hline 13 & Male & 44 & Hepatic lesions detected after 6 days of examinations & No \\
\hline 14 & Male & 65 & Hepatic lesions detected after $>2$ months of physical examinations & No \\
\hline 15 & Male & 49 & Diarrhea for $>$ I month; black stools for 5 days & Yes \\
\hline 16 & Male & 32 & Abdominal swelling for $>I$ month & No \\
\hline 17 & Male & 59 & Abdominal pain and swelling for $>I$ month & No \\
\hline 18 & Male & 66 & Abdominal pain and swelling for $>10$ days & No \\
\hline 19 & Male & 70 & $\begin{array}{l}\text { Abdominal swelling and hypodynamia for I week; a I-day physical } \\
\text { examination revealed hepatic lesions }\end{array}$ & No \\
\hline 20 & Male & 62 & Hepatic lesions detected after 7 days of physical examinations & No \\
\hline 21 & Male & 42 & Detected hepatic lesions after 2 days of physical examinations & No \\
\hline 22 & Male & 53 & Hepatic lesions detected after 10 days of physical examinations & No \\
\hline 23 & Male & 65 & Physical examination revealed right hepatic lesions & No \\
\hline 24 & Male & 44 & Hepatic lesions detected after 7 days of physical examinations & Mild \\
\hline 25 & Male & 32 & Abdominal pain and swelling for $>20$ days & No \\
\hline 26 & Male & 35 & Abdominal pain and swelling for $>10$ days & No \\
\hline 27 & Male & 64 & Hepatic lesions detected after 7 days of physical examinations & No \\
\hline 28 & Male & 63 & Hepatic lesions detected after 7 days of physical examinations & No \\
\hline 29 & Male & 55 & Right abdominal pain, swelling, nausea, and vomiting for $>25$ days & Mild \\
\hline 30 & Male & 51 & Hepatic lesions detected after 7 days of physical examinations & No \\
\hline 31 & Female & 40 & Abdominal pain and swelling for $>20$ days & No \\
\hline 32 & Female & 31 & Diarrhea for $>$ I month; black stools for 5 days & Yes \\
\hline 33 & Female & 49 & Abdominal pain and swelling for $>I$ month & No \\
\hline 34 & Female & 53 & Right abdominal pain, swelling, nausea, and vomiting for $>I$ month & Mild \\
\hline 35 & Female & 59 & Abdominal pain and swelling for $>I$ month & No \\
\hline 36 & Female & 61 & Diarrhea for $>$ I month; black stools for 5 days & Yes \\
\hline 37 & Female & 41 & Abdominal pain and swelling for $>I$ month & No \\
\hline 38 & Female & 67 & Hepatic lesions detected after 7 days of physical examinations & No \\
\hline 39 & Female & 58 & Abdominal pain and swelling for $>15$ days & No \\
\hline 40 & Female & 48 & Hepatic lesions detected after $>$ I month of physical examinations & No \\
\hline 41 & Female & 47 & Chest pain for 10 days & Yes \\
\hline 42 & Female & 36 & Abdominal pain and swelling for $>20$ days & No \\
\hline 43 & Female & 70 & Right abdominal pain, swelling, nausea, and vomiting for $>I$ month & Mild \\
\hline 44 & Female & 36 & Hepatic lesions detected after 14 days of physical examinations & No \\
\hline 45 & Female & 56 & Right abdominal pain, swelling, nausea, and vomiting for $>I$ month & Mild \\
\hline 46 & Female & 59 & Hepatic lesions detected after 7 days of physical examinations & No \\
\hline 47 & Female & 49 & Hepatic lesions detected after $>$ I month of physical examinations & No \\
\hline 48 & Female & 46 & Hepatic lesions detected after 20 days of physical examinations & No \\
\hline 49 & Female & 34 & $\begin{array}{l}\text { Hepatic lesions detected after }>1.5 \text { months of physical } \\
\text { examinations }\end{array}$ & No \\
\hline 50 & Female & 38 & Hepatic lesions detected after 5 days of physical examinations & No \\
\hline
\end{tabular}

Abbreviations: AFP, alpha-fetoprotein; HCC, hepatocellular carcinoma. 
Table 2 Blood biochemical characteristics of the AFP-negative HCC patients

\begin{tabular}{|c|c|c|c|c|c|c|c|c|}
\hline Patient & ALT & AST & GGT & ADA & TBA & TBIL & PT & Test for hepatitis B \\
\hline I & 40.1 & 20.0 & $64.7 \uparrow$ & 12.8 & 5.3 & 10.7 & 10.9 & $(-)$ \\
\hline 2 & 46.5 & $43.7 \uparrow$ & $69.4 \uparrow$ & $42.5 \uparrow$ & $47.7 \uparrow$ & 7.9 & 11.0 & Small three positive \\
\hline 3 & 23.4 & 25.0 & 11.7 & $26.4 \uparrow$ & 6.4 & 19.3 & 11.4 & Small three positive \\
\hline 4 & 44.5 & $50.1 \uparrow$ & $106.9 \uparrow$ & 20.7 & $44.4 \uparrow$ & 13.8 & 11.7 & Small three positive \\
\hline 5 & $63.3 \uparrow$ & $54.9 \uparrow$ & $83.9 \uparrow$ & 20.2 & $53.8 \uparrow$ & 7.5 & 11.0 & Small three positive \\
\hline 6 & 28.6 & 24.5 & $69.5 \uparrow$ & 13.8 & 2.8 & 8.8 & 10.1 & $(-)$ \\
\hline 7 & 25.0 & 19.5 & 51.8 & 11.0 & 5.1 & 7.8 & 10.3 & Great three positive \\
\hline 8 & $105.8 \uparrow$ & $72.8 \uparrow$ & $191.7 \uparrow$ & 18.7 & $49.4 \uparrow$ & $28.5 \uparrow$ & $13.3 \uparrow$ & Small three positive \\
\hline 9 & 22.0 & 13.5 & 26.6 & 20.5 & 2.0 & 20.5 & $12.3 \uparrow$ & Small three positive \\
\hline 10 & $374.2 \uparrow$ & $458.8 \uparrow$ & $95.6 \uparrow$ & 25.0 & 11.7 & 18.4 & 12.0 & Great three positive \\
\hline 11 & 14.2 & 21.6 & 15.0 & 18.6 & 11.8 & 19.2 & 10.8 & $(-)$ \\
\hline 12 & $63.8 \uparrow$ & $87.6 \uparrow$ & 19.6 & 18.1 & $16.9 \uparrow$ & $33.0 \uparrow$ & $16.6 \uparrow$ & Small three positive \\
\hline 13 & $65.1 \uparrow$ & $63.9 \uparrow$ & $66.3 \uparrow$ & $29.5 \uparrow$ & $16.2 \uparrow$ & 10.4 & 11.2 & Great three positive \\
\hline 14 & $67.0 \uparrow$ & $47.1 \uparrow$ & $257.5 \uparrow$ & 12.6 & 11.5 & 10.0 & 12.0 & Small three positive \\
\hline 15 & 49.0 & 38.0 & 47.0 & 11.8 & 9.7 & 8.6 & $13.5 \uparrow$ & Small three positive \\
\hline 16 & 15.7 & 23.3 & 10.8 & 8.8 & 1.8 & 7.6 & $9.7 \downarrow$ & $(-)$ \\
\hline 17 & 20.9 & $44.2 \uparrow$ & $80.7 \uparrow$ & 18.2 & 7.6 & 13.1 & 10.7 & $(-)$ \\
\hline 18 & 23.9 & 24.2 & 24.1 & 22.0 & 2.1 & 15.1 & 10.1 & $(-)$ \\
\hline 19 & 13.9 & 17.6 & 16.5 & 1.7 & 2.6 & $4.6 \downarrow$ & 11.9 & $(-)$ \\
\hline 20 & 41.0 & $47.8 \uparrow$ & $303.0 \uparrow$ & 17.3 & 5.2 & 16.6 & 10.6 & $(-)$ \\
\hline 21 & 32.2 & 25.2 & 101 & 20.0 & 10.0 & 21.1 & 10.0 & Small three positive \\
\hline 22 & 14.3 & $46.6 \uparrow$ & $52.2 \uparrow$ & 16.9 & 11.9 & $26.0 \uparrow$ & 11.0 & Great three positive \\
\hline 23 & $41.4 \uparrow$ & 16.4 & 43.1 & 9.3 & 9.8 & 14.7 & $12.7 \uparrow$ & Small three positive \\
\hline 24 & $55.9 \uparrow$ & 28.5 & 16.9 & 14.8 & 15.3 & 16.9 & $14.1 \uparrow$ & $(-)$ \\
\hline 25 & $60.4 \uparrow$ & $37.2 \uparrow$ & 18.8 & 32.0 & 1.8 & 13.0 & 10.1 & Small three positive \\
\hline 26 & 35.7 & $47.3 \uparrow$ & 32.3 & 10.0 & 5.4 & 18.1 & $8.7 \downarrow$ & Small three positive \\
\hline 27 & 30.6 & 25.7 & 31.9 & 6.5 & 9.0 & 9.6 & 9.9 & $(-)$ \\
\hline 28 & 28.8 & $35.8 \uparrow$ & 43.5 & 21.1 & 7.5 & $4.9 \downarrow$ & 11.5 & Small three positive \\
\hline 29 & 30.2 & $45.9 \uparrow$ & $54.4 \uparrow$ & 17.4 & 6.4 & 13.4 & 12.1 & Great three positive \\
\hline 30 & 15.3 & 24.0 & $62.8 \uparrow$ & 18.8 & 10.6 & 16.8 & 13.7 & Small three positive \\
\hline 31 & 10.7 & $36.4 \uparrow$ & 43.6 & 13.0 & 4.4 & 18.9 & 10.8 & Small three positive \\
\hline 32 & 15.1 & 16.3 & 41.7 & 12.4 & 6.8 & 19.2 & 9.9 & Small three positive \\
\hline 33 & 34.2 & $56.2 \uparrow$ & 21.6 & 17.3 & 9.7 & 10.4 & 10.3 & $(-)$ \\
\hline 34 & 39.5 & $46.1 \uparrow$ & 35.5 & 15.4 & $12.4 \uparrow$ & II.I & 11.9 & $(-)$ \\
\hline 35 & 15.2 & $67.3 \uparrow$ & 41.1 & 7.5 & 11.6 & 17.6 & $12.5 \uparrow$ & Small three positive \\
\hline 36 & 28.7 & $43.5 \uparrow$ & 10.3 & 11.0 & $20.1 \uparrow$ & 20.3 & 11.0 & Small three positive \\
\hline 37 & 19.3 & $52.4 \uparrow$ & 39.4 & 3.7 & 7.7 & 21.8 & 9.8 & Small three positive \\
\hline 38 & 20.7 & 16.6 & 28.7 & 9.9 & 6.6 & $25.1 \uparrow$ & 10.1 & $(-)$ \\
\hline 39 & 21.9 & 18.7 & $68.9 \uparrow$ & 5.8 & 2.3 & 16.4 & 9.9 & Small three positive \\
\hline 40 & 17.6 & 19.8 & $56.5 \uparrow$ & 30.0 & 1.4 & 10.9 & 10.1 & Small three positive \\
\hline 41 & 36.4 & 20.9 & 36.3 & 19.8 & 3.1 & 12.4 & 11.4 & $(-)$ \\
\hline 42 & $68.8 \uparrow$ & $64.9 \uparrow$ & 41.4 & 12.7 & 1.6 & 16.6 & 12.0 & $(-)$ \\
\hline 43 & 35.0 & 25.6 & 32.6 & 14.6 & 5.7 & 18.8 & 10.5 & $(-)$ \\
\hline 44 & $102.2 \uparrow$ & 23.4 & 69.5 & 25.0 & 6.6 & 17.0 & $16.1 \uparrow$ & $(-)$ \\
\hline 45 & 21.3 & 24.5 & 26.9 & 8.9 & $46.1 \uparrow$ & 16.4 & $8.8 \downarrow$ & Small three positive \\
\hline 46 & 38.4 & 14.6 & 18.4 & 24.3 & 5.7 & 7.7 & 11.7 & $(-)$ \\
\hline 47 & 25.5 & 16.4 & 27.3 & 16.4 & 4.3 & $3.6 \downarrow$ & $13.9 \uparrow$ & Small three positive \\
\hline 48 & 21.7 & 19.3 & $109.1 \uparrow$ & $30.0 \uparrow$ & 6.1 & 8.5 & 11.1 & Small three positive \\
\hline 49 & $64.8 \uparrow$ & 20.8 & $76.0 \uparrow$ & 18.8 & 7.9 & 16.5 & 10.0 & $(-)$ \\
\hline 50 & 11.9 & $36.7 \uparrow$ & $58.9 \uparrow$ & 17.9 & 4.7 & $38.0 \uparrow$ & 9.9 & Small three positive \\
\hline
\end{tabular}

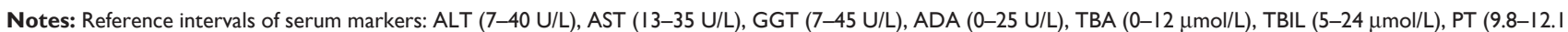
seconds), FB (I.8-3.5 g/L), APTT (22.7-3I.8 seconds), and TT (14-2I seconds). Tests for hepatitis B ( $\mathrm{HBsAg}, \mathrm{HBsAb}, \mathrm{HBeAg}, \mathrm{HBeAb}$, and $\mathrm{HBcAb})$ : small three positive (HBsAg-positive, $\mathrm{HBeAb}$-positive, and $\mathrm{HBcAb}$-positive); great three positive (HBsAg-positive, $\mathrm{HBeAg-positive,} \mathrm{and} \mathrm{HBcAb-positive).} \mathrm{(} \uparrow$ ), high level, ( $\downarrow$ ), low level.

Abbreviations: AFP, alpha-fetoprotein; $\mathrm{HCC}$, hepatocellular carcinoma; $\mathrm{HBs} A$ g, hepatitis $B$ surface antigen; $\mathrm{HBsAb}$, hepatitis $B$ surface antibody; $\mathrm{HBeAg}$, hepatitis $B$ e antigen; HBeAb, hepatitis B e antibody; HBcAb, hepatitis B core antibody; FB, fibrinogen; APTT, activated partial thromboplastin time; TT, thrombin time; ALT, alanine aminotransferase; AST, aspartate aminotransferase; GGT, gamma-glutamyl transpeptidase; ADA, adenosine deaminase; TBA, total bile acids; TBIL, total bilirubin; PT, prothrombin time. 


\section{Results}

\section{Comparison of AFP-L3 in different groups}

An affinity adsorption method was used to determine the serum level of AFP-L3 in all enrolled subjects. Among the 50 serum samples obtained from patients with a hepatocellular neoplasm, 25 were positive for AFP-L3. However, among the 30 samples obtained from patients with other liver diseases and 50 samples obtained from healthy subjects, only one sample from each group was positive for AFP-L3. Subjects with AFP-negative HCC had significantly higher serum levels of AFP-L3 compared to both non-HCC and healthy subjects $(P<0.01)$, while there was no significant difference in AFP-L3 levels between non-HCC patients and healthy subjects $(P>0.05)$. The outcomes of this analysis are shown in Table 3.

\section{Comparison of GP73 in different groups}

The GP73-positive rates among patients with AFP-negative liver cancer, patients without liver cancer, and healthy subjects were $66 \%, 10 \%$, and $0 \%$, respectively. While patients with liver cancer had significantly higher serum levels of GP73 compared to non-HCC patients and healthy subjects, there was no significant difference in GP73 serum levels between non-HCC patients and healthy subjects. A comparison of GP73-positive rates in the three groups is shown in Table 4.

\section{Diagnostic utility of AFP-L3 and GP73}

In the ROC curve analysis, the diagnostic accuracy of AFP-L3 and GP73 was evaluated. The maximum area under the curve for AFP-L3 in distinguishing AFP-negative HCC was 0.6094 (95\% confidence interval, 0.5992-0.7996), and the sensitivity, specificity, and accuracy were 50\%, $97.5 \%$, and $79.2 \%$, respectively. For GP73, the maximum area under the curve was 0.7811 (95\% confidence interval, $0.7581-0.9241)$, and the sensitivity, specificity, and accuracy were $66 \%, 96.2 \%$, and $84.6 \%$, respectively. ROC curves for

Table 3 Comparison of AFP-L3-positive rates in the three AFP-negative groups

\begin{tabular}{llllll}
\hline Group & Patient & \multicolumn{2}{l}{ AFP-L3 } & $\chi^{2}$ & P-value \\
\cline { 3 - 5 } & & $\begin{array}{l}\text { Positive } \\
\text { cases }\end{array}$ & $\begin{array}{l}\text { Positive } \\
\text { rate (\%) }\end{array}$ & \\
\hline HCC group & 50 & 25 & 50.0 & $9.35^{\mathrm{a}}$ & $0.002^{\mathrm{a}}$ \\
Non-HCC group & 30 & $\mathrm{I}$ & 3.3 & $13.28^{\mathrm{b}}$ & $0.003^{\mathrm{b}}$ \\
Healthy group & 50 & $\mathrm{I}$ & 2.0 & $0.14^{\mathrm{c}}$ & $0.032^{\mathrm{c}}$ \\
\hline
\end{tabular}

Notes: a $\mathrm{HCC}$ group compared with non-HCC group; ${ }^{b} \mathrm{HCC}$ group compared with healthy group; 'non-HCC group compared with healthy group.

Abbreviations: AFP, alpha-fetoprotein; HCC, hepatocellular carcinoma.
Table 4 Comparison of GP73-positive rates in the three AFP-negative groups

\begin{tabular}{llllll}
\hline Group & Patient & \multicolumn{2}{l}{ GP73 } & $\chi^{2}$ & \multirow{2}{*}{ P-value } \\
\cline { 3 - 5 } & & $\begin{array}{l}\text { Positive } \\
\text { cases }\end{array}$ & $\begin{array}{l}\text { Positive } \\
\text { rate (\%) }\end{array}$ & \\
\hline HCC group & 50 & 33 & $66(33 / 50)$ & $8.25^{\mathrm{a}}$ & $0.009^{\mathrm{a}}$ \\
Non-HCC group & 30 & 3 & $10(3 / 30)$ & $23.35^{\mathrm{b}}$ & $0.002^{\mathrm{b}}$ \\
Healthy group & 50 & 0 & 0 & $2.47^{\mathrm{c}}$ & $0.036^{\mathrm{c}}$ \\
\hline
\end{tabular}

Notes: a HCC group compared with non-HCC group; ${ }^{b} \mathrm{HCC}$ group compared with healthy group; 'non-HCC group compared with healthy group.

Abbreviations: AFP, alpha-fetoprotein; GP73, Golgi protein 73; HCC, hepatocellular carcinoma.

the diagnosis of HCC using AFP-L3 and GP73 are shown in Figure 1 and the statistical outcomes in Table 5.

\section{Diagnostic utility of combined AFP-L3 and GP73 tests}

If an assay for either AFP-L3 or GP73 showed a positive result, the outcome of the diagnosis was classified as positive. Among the 50 serum samples obtained from patients with a hepatocellular neoplasm, 20 samples were positive for both AFP-L3 and GP73, five samples were positive for AFP-L3 but negative for GP73, and ten were positive for GP73 but negative for AFP-L3. None of the 30 samples obtained from patients with other liver diseases were positive for both AFP-L3 and GP73; however, one sample was positive for AFP-L3 but negative for GP73, and one sample was positive for GP73 but negative for AFP-L3. Among the 50 samples obtained from healthy subjects, none were positive for both AFP-L3 and GP73, one sample was positive for AFP-L3 but

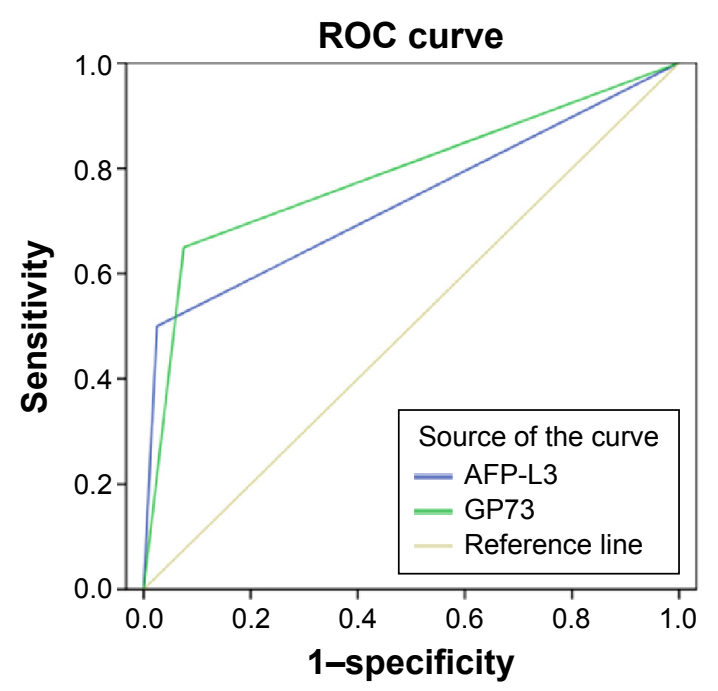

Figure I ROC curves of AFP-L3 or GP73 levels for the diagnosis of HCC. Abbreviations: AFP, alpha-fetoprotein; GP73, Golgi protein 73; HCC, hepatocellular carcinoma; ROC, receiver operator characteristic. 
Table 5 The performance of individual AFP-L3 and GP73 tests in diagnosing AFP-negative HCC

\begin{tabular}{llll}
\hline $\begin{array}{l}\text { Detection } \\
\text { model }\end{array}$ & Sensitivity (\%) & Specificity (\%) & Accuracy (\%) \\
\hline AFP-L3 & $50.0(25 / 50)$ & $97.5(78 / 80)$ & $79.2(103 / 130)$ \\
GP73 & $66.0(33 / 50)$ & $96.2(77 / 80)$ & $84.6(110 / 130)$ \\
\hline
\end{tabular}

Abbreviations: AFP, alpha-fetoprotein; GP73, Golgi protein 73; HCC, hepatocellular carcinoma.

negative for GP73, and no sample was positive for GP73 but negative for AFP-L3. The sensitivity, specificity, and accuracy achieved when using combined AFP-L3 plus GP73 assay results were $40 \%, 100 \%$, and $76.9 \%$, respectively. The outcomes of these analyses are shown in Table 6.

\section{Discussion}

A clinical diagnosis of HCC is easy if based on significantly increased serum AFP levels combined with definitive imaging results. However, AFP-negative HCC is not as easily diagnosed and becomes the focus of this study. A diagnosis of AFP-negative HCC depends largely on imaging results, which often shows defects such as a missed focus of disease in the right posterior portion of the liver adjoining the diaphragmatic dome by $\mathrm{B}$ ultrasound due to interference from air in the lung. Additionally, due to its high expense, a CT scan cannot be repeatedly performed in a short period. In recent years, numerous studies have been conducted to identify a diagnostic biomarker for AFP-negative HCC; however, all of the potential candidates have shown poor specificity and sensitivity. The clinical characteristics of AFP-negative HCC are as follows: 1) abdominal pain is the most common local symptom and manifests as a dull or stabbing pain in the right upper quadrant; 2) the abdominal pain can be intermittent or continuous; and 3) the pain is much severer at night and may radiate to the right shoulder and back. In this study, $50 \%$ of the subjects had abdominal pain. Our cohort of AFP-negative HCC patients included 17 males and three females thus supporting the notion

Table 6 The performance of combined AFP-L3 and GP73 assays for diagnosing AFP-negative HCC

\begin{tabular}{llll}
\hline Detection model & $\begin{array}{l}\text { Sensitivity } \\
(\%)\end{array}$ & $\begin{array}{l}\text { Specificity } \\
(\%)\end{array}$ & $\begin{array}{l}\text { Accuracy } \\
(\%)\end{array}$ \\
\hline AFP-L3 (+) and GP73 (+) & $40.0(20 / 50)$ & $100(80 / 80)$ & $76.9(100 / 130)$ \\
AFP-L3 (+) and GP73 (-) & $10.0(5 / 50)$ & $98.8(79 / 80)$ & $64.6(84 / 130)$ \\
AFP-L3 (-) and GP73 (+) & $20.0(10 / 50)$ & $98.8(79 / 80)$ & $68.5(89 / 130)$ \\
AFP-L3 (-) and GP73 (-) & $6.0(3 / 50)$ & $93.7(75 / 80)$ & $60.0(78 / 130)$ \\
\hline
\end{tabular}

Abbreviations: AFP, alpha-fetoprotein; GP73, Golgi protein 73; HCC, hepatocellular carcinoma. described in previous reports that AFP-negative $\mathrm{HCC}$ is more prevalent in males than females. ${ }^{14-16}$ This phenomenon might be mainly caused by higher rates of smoking and drinking among males. ${ }^{14}$ Ogden et al ${ }^{17}$ proposed that the $\mathrm{HBx}$ protein in HBV combines with $\mathrm{p} 53$ to alleviate $\mathrm{p} 53$-mediated repression of AFP gene expression, leading to overexpression of AFP. In our current study, up to $65 \%$ of AFP-negative HCC patients were infected with $\mathrm{HBV}$, indicating AFP expression is influenced by a variety of factors. Izumi et $\mathrm{al}^{18}$ found that AFP-negative HCC patients had a significantly higher 5-year survival rate compared to AFP-positive patients. Additionally, the rates of positive rate for gamma-glutamyl transpeptidase (GGT), alanine aminotransferase (ALT), aspartate aminotransferase (AST), adenosine deaminase (ADA), total bile acids (TBA), total bilirubin (TBIL), and prothrombin time (PT) in AFP-negative HCC patients were $45 \%, 30 \%, 50 \%, 15 \%, 30 \%, 10 \%$, and $20 \%$, respectively, and were notably lower than those in AFP-positive HCC patients indicating that values for GGT, ALT, AST, ADA, TBA, TBIL, and PT are not highly specific for identifying individuals with AFP-negative HCC.

GP73 is an early marker of $\mathrm{HCC}^{12}$ and is highly expressed only in individuals with acute or chronic liver disease, liver cirrhosis, or HCC. GP73 is thus regarded as a highly sensitive and specific biomarker for liver disease. When setting the critical value for AFP-L3 at 5\% for AFP-negative patients (ie, $<20 \mu \mathrm{g} / \mathrm{L}$ indicating $\mathrm{HCC}$ ), Toyoda et $\mathrm{al}^{19}$ found that AFP-L3 had $41.5 \%$ specificity and $85.1 \%$ sensitivity for diagnosing HCC. Moreover, AFP-L3 levels were found to be independent of total AFP levels, suggesting AFP-L3 as an independent diagnostic index, which is unrelated to levels of total AFP. When used for diagnosing HCC, AFP-L3 has higher specificity than total AFP, but similar sensitivity. ${ }^{20}$ While total serum AFP can be used to diagnose $\sim 70 \%$ of HCC cases, $\sim 30 \%$ of HCC cases are AFP-negative, ${ }^{21,22}$ leading to their missed or misdiagnosis. Thus, the accuracy of HCC diagnoses has been obviously increased by assessing multiple biomarkers.

Our results from evaluating serum levels of AFP-L3 and GP73 in the current study support the following conclusions: 1) compared with the non-HCC and healthy groups, the AFP-negative HCC group had a significantly higher percentage of individuals who tested positive for both AFP-L3 and GP73;2) the sensitivity, specificity, and accuracy of AFP-L3 for diagnosing AFP-negative HCC were 50.0\%, $97.5 \%$, and $79.2 \%$, respectively, while those of GP73 were $66.0 \%, 96.2 \%$, and $84.6 \%$, respectively; and 3) when using combined AFP-L3 and GP73 assay results to diagnosis 
AFP-negative HCC, the sensitivity, specificity, and accuracy were $40.0 \%, 100.0 \%$, and $76.9 \%$, respectively. Thus, sensitivity and accuracy achieved when using combined AFP-L3 and GP73 assay to diagnose AFP-negative HCC were higher than those achieved when using either assay result alone. Based on these data, we recommend using the combined assay for diagnosing HCC, as this method can aid in making an early, accurate, and differential diagnosis of AFP-negative HCC. A limitation of our study was that it included only 50 AFP-negative HCC patients because of low occurrence rate of $\mathrm{HCC}$, and the etiology of $\mathrm{HCC}$ in Chinese population should also be considered, as it may not be the same as in other countries such as Japan, and the USA. Clinical trials with not only larger sample sizes and which include patient follow-up procedures but also populations from distinct regions must be conducted to confirm the value of using a combination of AFP-L3 and GP73 assay results for diagnosing AFP-negative HCC.

\section{Acknowledgment}

This study was supported by grants from the projects of medical and health technology development program in Shandong Province (no 2011HW063).

\section{Disclosure}

The authors report no conflicts of interest in this work.

\section{References}

1. Jemal A, Siegel R, Xu J, Ward E. Cancer statistics. CA Cancer J Clin. 2010;60(5):277-300.

2. Shariff MI, Cox IJ, Gomaa AI, Khan SA, Gedroyc W, Taylor-Robinson SD. Hepatocellular carcinoma: current trends in worldwide epidemiology, risk factors, diagnosis and therapeutics. Expert Rev Gastroenterol Hepatol. 2009;3(4):353-367.

3. Mao Y, Yang H, Xu H, et al. Golgi protein 73 (GOLPH2) is a valuable serum marker for hepatocellular carcinoma. Gut. 2010;59(12):1687-1693.

4. Xu WJ, Guo BL, Han YG, Shi L, Ma WS. Diagnostic value of alphafetoprotein-L3 and Golgi protein 73 in hepatocellular carcinomas with low AFP levels. Tumour Biol. 2014;35(12):12069-12074.

5. Gao J, Xu AF, Zheng HY, Chen G, Wang MC, Lou GQ. [Clinical application studies on AFP-L3 detected by micro-spin column method]. Zhonghua Shi Yan He Lin Chuang Bing Du Xue Za Zhi. 2010;24(6):461-463.
6. Kumada T, Toyoda H, Kiriyama S, et al. Predictive value of tumor markers for hepatocarcinogenesis in patients with hepatitis $\mathrm{C}$ virus. J Gastroenterol. 2011;46(4):536-544.

7. Shiraki K, Takase K, Tameda Y, Hamada M, Kosaka Y, Nakano T. A clinical study of lectin-reactive alpha-fetoprotein as an early indicator of hepatocellular carcinoma in the follow-up of cirrhotic patients. Hepatology. 1995;22(3):802-807.

8. Kumada T, Nakano S, Takeda I, et al. Clinical utility of Lens culinaris agglutinin-reactive alpha-fetoprotein in small hepatocellular carcinoma: special reference to imaging diagnosis. J Hepatol. 1999;30(1): $125-130$.

9. Kladney RD, Bulla GA, Guo L, et al. GP73, a novel Golgi-localized protein upregulated by viral infection. Gene. 2000;249(1-2):53-65.

10. Kladney RD, Cui X, Bulla GA, Brunt EM, Fimmel CJ. Expression of GP73, a resident Golgi membrane protein, in viral and nonviral liver disease. Hepatology. 2002;35(6):1431-1440.

11. Block TM, Comunale MA, Lowman M, et al. Use of targeted glycoproteomics to identify serum glycoproteins that correlate with liver cancer in woodchucks and humans. Proc Natl Acad Sci U S A. 2005;102(3): 779-784.

12. Marrero JA, Romano PR, Nikolaeva O, et al. GP73, a resident Golgi glycoprotein, is a novel serum marker for hepatocellular carcinoma. J Hepatol. 2005;43(6):1007-1012.

13. Tian L, Wang Y, Xu D, et al. Serological AFP/Golgi protein 73 could be a new diagnostic parameter of hepatic diseases. Int J Cancer. 2011; 129(8):1923-1931.

14. Lai CL, Gregory PB, Wu PC, Lok AS, Wong KP, Ng MM. Hepatocellular carcinoma in Chinese males and females. Possible causes for the male predominance. Cancer. 1987;60(5):1107-1110.

15. Yang D, Hanna DL, Usher J, et al. Impact of sex on the survival of patients with hepatocellular carcinoma: a surveillance, epidemiology, and end results analysis. Cancer. 2014;120(23):3707-3716.

16. Xue TC, Zhang L, Ren ZG, et al. Sex-determination gene SRY potentially associates with poor prognosis but not sex bias in hepatocellular carcinoma. Dig Dis Sci. 2015;60(2):427-435.

17. Ogden SK, Lee KC, Barton MC. Hepatitis B viral transactivator $\mathrm{HBx}$ alleviates p53-mediated repression of alpha-fetoprotein gene expression. J Biol Chem. 2000;275(36):27806-27814.

18. Izumi R, Shimizu K, Kiriyama M, et al. Alpha-fetoprotein production by hepatocellular carcinoma is prognostic of poor patient survival. J Surg Oncol. 1992;49(3):151-155.

19. Toyoda H, Kumada T, Tada T, et al. Clinical utility of highly sensitive Lens culinaris agglutinin-reactive alpha-fetoprotein in hepatocellular carcinoma patients with alpha-fetoprotein $<20 \mathrm{ng} / \mathrm{mL}$. Can Sci. 2011; 102(5):1025-1031.

20. Yoon SK. Recent advances in tumor markers of human hepatocellular carcinoma. Intervirology. 2008;51(Suppl 1):34-41.

21. Wudel LJ Jr, Chapman WC. Indications and limitations of liver transplantation for hepatocellular carcinoma. Surg Oncol Clin N Am. 2003(1);12:77-90.

22. Soresi M, Magliarisi C, Campagna P, et al. Usefulness of alpha-fetoprotein in the diagnosis of hepatocellular carcinoma. Anticancer Res. 2003; 23(2c):1747-1753.
OncoTargets and Therapy

\section{Publish your work in this journal}

OncoTargets and Therapy is an international, peer-reviewed, open access journal focusing on the pathological basis of all cancers, potential targets for therapy and treatment protocols employed to improve the management of cancer patients. The journal also focuses on the impact of management programs and new therapeutic agents and protocols on

\section{Dovepress}

patient perspectives such as quality of life, adherence and satisfaction. The manuscript management system is completely online and includes a very quick and fair peer-review system, which is all easy to use. Visit http://www.dovepress.com/testimonials.php to read real quotes from published authors. 